Sood A S

Bhardwaj B

Gill J S

Department of ENT

Sri Guru Ram Das Institute of Medical

Sciences \& Research (SGRDIMSR)

Amritsar, India

Correspondence to:

Dr. Arvinder Singh Sood

Associate Professor

22A, Rajinder Nagar

Opposite Dhingra Hospital

Sultanwind Road

Amritsar- 143001

e-mail:drarvindersinghsood@gmail.com

\section{PRIMARY TUBERCULOSIS OF THE TONSIL}

Primary tuberculosis of tonsil is a rare clinical entity. This entity is often misdiagnosed as its presentation mimicks malignancy. This case report describes a case of primary tuberculosis of tonsil which was simulating malignancy. There are certain clinical pointers which if kept in mind can hint towards the diagnosis of this disease. Treatment is medical with excellent cure rate.

Key words : tuberculosis, tonsil, sore throat, cervical lymphadenopathy
INTRODUCTION:

Tuberculosis in humans is caused by Mycobacterium tuberculosis and Mycobacterium bovis. Though tuberculosis is an omnipresent disease, it is a great simulator. Its variable clinical presentations often let diagnosis slip out of clinicians hand. Incidence of tuberculosis in lungs is as high as $98 \% .^{1}$ Despite the contiguity of mucosa and contact of pulmonary secretions with palatine tonsils, the incidence of tuberculosis in tonsils is less than $5 \% .^{2}$ Incidence of primary tuberculosis of tonsil which without associated pulmonary tuberculosis is very rare. ${ }^{3}$ Tuberculosis of tonsil is still a diagnostic challenge for an otolaryngologist as it is commonly misdiagnosed. Early diagnosis is essential as the treatment is medical and outcomes are favourable. We present a case of primary tuberculosis of tonsil which was suspected as malignancy. We believe it to be of interest because of the rarity of the condition and the diagnostic problem it presents.

CASE REPORT:

A 58 year old female presented in our department with complaints of stiffness of mouth and dull aching pain in throat for two and half months duration. She also had 2-3 episodes of fresh bleeding from oral cavity during this period. Though the bleed was small in amount and stopped by itself, it was typically preceded by minor trauma as during or after brushing. On examining the oral cavity and oropharynx, an irregular proliferative mass was seen hanging down from the upper pole of right tonsil and rest of the tonsillar mass was hypertrophied. On the left side, though no well defined lesion was seen but tonsillar remnants were irregular and thickened. Indirect laryngoscopy was

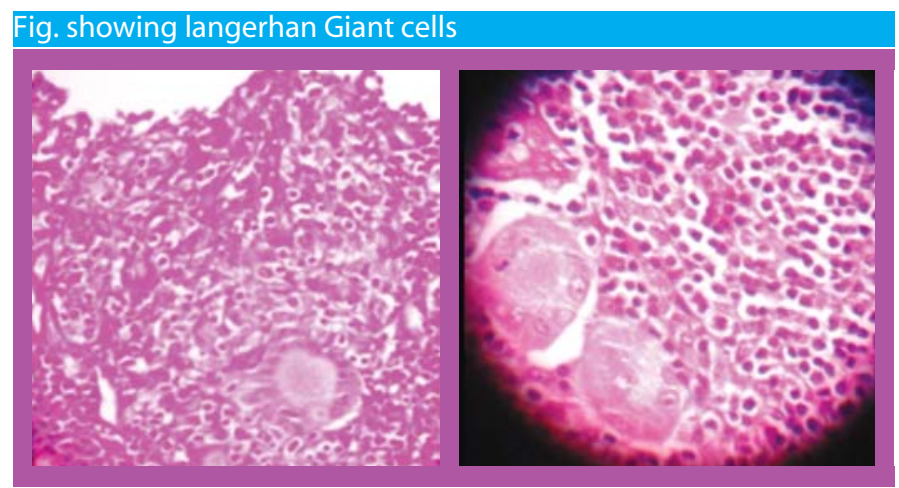

performed and it was found that the mass extended beyond the lower limit of the lower pole. Both tonsillar area were firm to hard on palpation, but more on right side. Overlying mucosa were thickened and fibrotic. Base of tongue was soft. Neck nodes were not palpable. Nasal examination revealed a mild left sided deviated nasal septum with inferior turbinate hypertrophy on same side. Ear examination was normal. To rule out malignancy, CT scan was done, which showed a polypoidal lesion in relation to oropharynx on right side superior to epiglottis involving the pharyngoepiglottic fold on the same side. CT scan also documented associated lymphadenopathy along jugular chain in the neck bilaterally. The findings on CT scan consolidated suspicion of malignancy. A punch biopsy from the mass was taken and the histopathological examination report suggested inflammatory pathology with epithelial atypia. 'Epithelial atypia' was perse a dangerous signal. To benefit the patient and satisfy our ethical instinct we decided to completely remove the remaining mass along with tonsils bilaterally.Tonsillectomy was done under general anaesthesia by blunt dissection and snare technique. Complete specimen was sent for histopathological examination. Patient was followed up at weekly intervals and there was some alleviation in her symptoms. Histopathological report that followed 10 days later documented necrotizing granulomatous lesion suggestive of tubercular tonsillitis. The patient was referred to Chest and TB department with report to get an expert opinion and to get evaluated for pulmonary tuberculosis as well. Chest X-ray didn't show any signs of pulmonary tuberculosis and sputum for AFB was negative. The case was categorised as Primary tuberculosis of tonsil and the treatment was started. Patient is currently under treatment and her symptoms have completely subsided.

\section{DISCUSSION:}

Diagnosis of tuberculosis of tonsil is of high degree of suspicion. In this era of advanced medical and surgical treatment where tuberculosis is curable, losing a patient to tuberculosis due to delay in diagnosis is not justifiable. Predisposing factors for primary oral tuberculosis include poor dental hygiene, dental extraction, periodontitis and leucoplakia. It has been postulated that such infections are acquired by inhalation, with harbouring of disease in Waldeyer's ring. 4-5 The most common presentation includes sore throat, painful ulcerations, and cervical lymphadenopathy. These findings along with abnormal tonsils make a clinician highly suspicious of malignancy and differentiation from it becomes difficult. 6 More so, the changing 
epidemiological factors like advent of pasteurization techniques have decreased the incidence of primary tuberculosis of tonsil further, whereas the incidence of malignancy perse is on rise. The final diagnosis of tubercular tonsillitis is based on histopathological findings of the tonsillectomy material. ${ }^{7}$ Howsoever there are certain points referred to as Arbol's pointers which hint towards this entity. ${ }^{2}$ These include:

Unexplained granular or asymmetric enlargement of tonsil

- Tonsillar enlargement without exudates

- Obliteration of crypts

$\rightarrow$ Painful deglutition

MPresence of enlarged mobile jugulodigastric nodes

The suspicious lesions should always be sent for biopsy. Patients having biopsy positive for tonsillar tuberculosis should get a chest $\mathrm{x}$-ray done to rule out pulmonary foci. In the presence of lesions in lung, the tuberculosis of tonsil is considered secondary and if absent, the entity is labelled as primary. ${ }^{2}$ The treatment is in form of anti-tuberculosis therapy. ${ }^{8}$ The results are favourable.

\section{CONCLUSION:}

We should all come to the fact that tuberculosis of tonsil still exists. As an otolaryngologist we are the first resorts of patients with complaints pertaining to oral cavity and should always be alert to the possible diagnosis of tuberculosis of tonsil.

\section{REFERENCES}

1 Chavolla R, Dolci GF, Hernandez JFM etal. Primary tuberculosis of tonsil. International Journal Of Paediatric Otorhinolaryngology Extra. 2006, 1(2) ;150-3

2 Balasubramanian T. Tonsillar Tuberculosis : a literature review. Otolaryngology online.

3 Kant S, Verma SK, Sanjay.Isolated tonsil tuberculosis. Lung India. 2008, 25(4) ; 163-4.

4 Selimoglu E, Sutbeyaz Y, Ciftcioglu A, et al. Primary tonsillar tuberculosis: a case report. J LaryngolOtol 1995;109: 880-2.

5 Pedrol E, Estruch R, Barcelo J,Urbano Marquez A. Tonsillar and pharyngeal tuberculosis in a patient without HIV antibodies. J Infect Dis. 1989, 159(3); 598

6 Srirompotong S, Yimtae K, Srirompotong S. Clinical aspects of tonsillar tuberculosis. Southeast Asian J Trop Med PublicHealth. 2002 , 33(1); 147-50.

7 Celik O,Yakin S, Haner A, Celik P, Ozecian R.Tuberculous Tonsillitis. J Otolaryngology 1995 , 24(5) ; 307-9. 